\title{
La economía del grupo doméstico: determinante de muerte materna entre mujeres indígenas de Chiapas, México
}

\author{
María del Carmen Herrera Torres, ${ }^{1}$ Jorge Luis Cruz Burguete, ${ }^{2}$ \\ Gabriela Patricia Robledo Hernández ${ }^{3}$ y Guillermo Montoya Gómez ${ }^{2}$
}

Forma de citar Herrera Torres MC, Cruz Burguete JL, Robledo Hernández GP, Montoya Gómez G. La economía del grupo doméstico: determinante de muerte materna entre mujeres indígenas de Chiapas, México. Rev Panam Salud Publica. 2006;19(2):69-78.

RESUMEN Objetivo. Evaluar la situación económica y las relaciones de género en cuanto factores determinantes de muerte materna en la subregión de Patwitz, en Chiapas, México.

Métodos. Se realizó un estudio cuantitativo y cualitativo mediante la aplicación de una encuesta a 158 familias, entrevistas a profundidad a personas vinculadas con los casos de muerte materna y a líderes sociales de la región, así como grupos focales con parteras.

Resultados. La toma de decisiones respecto a la salud de la mujer en el seno de la familia constituye un problema crítico por cuanto depende exclusivamente del esposo y los familiares de este. Cuando el embarazo o el parto conllevan algún riesgo, tal situación introduce restricciones en la elección de opciones de atención fuera de la comunidad, de modo que 48,7\% de los casos obstétricos son atendidos por parteras, $45,3 \%$ por familiares y $6 \%$ por la pareja. El problema se agrava por la alta marginación y el muy bajo indice de desarrollo humano que caracterizan a la región estudiada, por la falta de acceso de las mujeres a la posesión de bienes, incluida la herencia de la tierra, y por el hecho de que 97,7\% de ellas hablan solamente lenguas indígenas.

Conclusiones. Las inequidades por razones de género en las familias indígenas, aunadas a una economía que no cubre las necesidades básicas, son factores que contribuyen a que las mujeres no reciban atención en el proceso reproductivo. Debido al bajo nivel socioeconómico, las decisiones que se toman en torno a la atención del embarazo, el parto y el puerperio tienen un alto costo en la salud y en la vida de las mujeres.

Palabras clave Mortalidad materna, economía, identidad de género, matronas, México.

Programa de Maestría, El Colegio de la Frontera Sur (ECOSUR), San Cristóbal de Las Casas, Chiapas, México. Toda correspondencia deberá dirigirse a María del Carmen Herrera Torres, El Colegio de la Frontera Sur (ECOSUR), Carretera Panamericana y Periférico Sur s/n., CP 29290, San Cristóbal de Las Casas, Chiapas, México. Teléfono (+52) (967) 67800 97. Correo electrónico: carmelita 630729@hotmail.com

2 El Colegio de la Frontera Sur (ECOSUR), San Cristóbal de Las Casas, Chiapas, México.

3 Centro de Investigaciones y Estudios Superiores en Antropología Social (CIESAS)-Sureste, San Cristóbal de Las Casas, Chiapas, México.
La Organización Panamericana de la Salud (OPS) estima que cada cinco minutos muere una mujer por complicaciones del embarazo, parto, puerperio o aborto, y que muchas que sobreviven pueden padecer secuelas por el resto de su vida, particularmente en los países pobres $(1,2)$.

Los acuerdos internacionales que han integrado a sus objetivos la reduc- ción de las muertes maternas, ${ }^{4}$ como el de la Conferencia Internacional de Población y Desarrollo (CIPD) en 1994 y

\footnotetext{
La Organización Mundial de la Salud (OMS) define la muerte materna como el fallecimiento de una mujer durante el embarazo o en los 42 días que siguen al término, independientemente del tiempo o el lugar en que ocurra, por cualquier causa relacionada con él o agravada por él o por su atención, pero no por causas accidentales o incidentales (3).
} 
el de la Conferencia Internacional de la Mujer en 1995, no han tenido una aplicación coherente en México, en Chiapas en particular, y están amenazados por la falta de políticas públicas encaminadas a corregir las desigualdades. Según los datos del informe de gobierno correspondiente al año 2000, los presupuestos estatales destinados a los programas de salud de la mujer fueron del orden de $0,003 \%$ del total y para el año 2001 el incremento fue mínimo, a $0,11 \%$; es decir, el presupuesto designado para la salud de la mujer se ha hecho visible, pero no sustancial. La falta de recursos públicos debilita el acceso y la calidad de los servicios en las regiones más necesitadas $(4,5)$.

El impacto de la política pública errática se resiente más en entidades como Chiapas, donde 30,63\% de la población de los 118 municipios que integran el estado tiene un índice de marginación bajo o muy bajo, mientras que la tercera parte de los municipios indígenas se clasifican en el nivel de muy alta marginación. ${ }^{5}$

En el decenio de 1980 el producto interno bruto (PIB) agrícola se consideró un pilar de la economía nacional de México, pero la caída vertiginosa de este sector en todo el país hizo crisis en Chiapas en 1994, con el surgimiento del movimiento armado zapatista y sus demandas de atención económica, social y cultural, que pusieron en primer plano las graves carencias de la población. En la mayor parte de los municipios de Chiapas las actividades económicas más importantes seguían siendo la agropecuaria y la silvícola, lo que incrementó la demanda de recursos en el área, en forma de subsidios para programas de apoyo al campo, en particular para el cultivo del maíz, un producto de consumo básico en los municipios marginados, seguido de algún cultivo comercial destinado a insertarse en los mercados internaciona-

\footnotetext{
Para este denominado Índice Chiapaneco de Marginación se consideraron cuatro dimensiones: a) porcentaje de población que no tiene acceso a vivienda digna, b) alcances del sistema educativo, c) ingresos monetarios y d) condiciones de dispersión geográfica. (5).
}

les, como es el caso del café. A pesar de que la región se considera importante productora de café, la entidad registra un bajo índice de desarrollo humano (IDH).

En este contexto, cada año ocurren 80 muertes maternas, que se concentran principalmente en los municipios más marginados de las regiones Sierra y Altos. En esta última se encuentra la subregión Patwitz, municipio de San Juan Cancuc, que fue el foco de la presente investigación. En la región Altos, más de la tercera parte de los municipios son indígenas, de muy alta marginación y con un bajo IDH (6). Se estima en $45 \%$ el subregistro de muertes maternas en la región Altos, lo cual significa que cada año podrían estar presentándose más de los 20 casos que se registran en promedio (4).

San Juan Cancuc, que fue declarado municipio libre en 1991, cumple con estos indicadores. Esta zona representa $0,21 \%$ del área de superficie estatal, con una población de 20688 personas, de las cuales $90,04 \%$ son hablantes de la lengua indígena tseltal y más de la tercera parte son monolingües (7).

En este municipio la mortalidad femenina excedente en el grupo de 15 a 49 años es la más alta de la región y del estado. La autora principal infiere (8) que muchas defunciones se clasificarían como muertes maternas. ${ }^{6}$ Los registros del Instituto de Salud en Chiapas (ISECH) lo clasifican como área de alta prioridad por la repetición de casos de muerte durante más de cinco años.

Este municipio se caracteriza por estar dividido por una montaña, que lo separa en dos subregiones con diferentes grados de desarrollo. Una es la denominada Centro Cancuc, que tiene $78,68 \%$ de la población total. En esta zona está situada la cabecera municipal, centro político administrativo, lo que contribuye a que las localidades tengan mayor acceso a los servicios bá-

\footnotetext{
6 El riesgo de morir entre las mujeres de este municipio es nueve veces mayor que el de sus pares nacionales y tres veces mayor que el de sus pares estatales. Si bien la razón de mortalidad de varón a mujer es de 1:1, debe considerarse que la mortalidad a nivel nacional es menor en mujeres del grupo de edad de 15 a 49 años (8).
}

sicos de abastecimiento de agua potable y luz eléctrica.

La otra es la subregión Patwitz, que concentra solo $21,32 \%$ de la población, distribuida en nueve comunidades, $y$ está habitada por 5462 personas, con una alta densidad demográfica y con servicios públicos deficientes. Esta pequeña franja de tierra comparte lazos culturales y sociales con el resto del municipio. Se comunica con el exterior por una carretera sin pavimentar que se dirige al municipio de Pantelhó, cabecera municipal que funciona como centro de intercambio comercial. Los ingresos de la subregión dependen básicamente de la comercialización del café; debido a la limitada capacidad para aprovechar la tierra, los cultivos de maíz y frijol (judía, poroto) resultan incosteables y poco productivos (figura 1).

La elección de Patwitz como foco de la presente investigación se debió a que las características señaladas crean el entramado en que ocurre cada año más de una muerte materna por complicaciones del embarazo, parto, puerperio o aborto. No obstante el evidente exceso de mortalidad femenina, parece haber todavía un subregistro de las muertes maternas, dada la costumbre de enterrar a las muertas en las parcelas. Además de suponer algún valor tradicional, esta práctica permite soslayar implicaciones morales y sociales, lo que a menudo lleva a los familiares a falsear la causa de la muerte en su declaración a las autoridades locales.

En torno a la región Altos se han publicado estudios de mortalidad materna $(4,9-11)$. El trabajo de investigación realizado en los municipios de Chenalhó, Larráinzar, San Cristóbal y como parte del proyecto Disminución de la Mortalidad Materna para la Región Altos (4) adoptó un enfoque sociocultural y antropológico. Entre sus principales hallazgos destacan los factores culturales que intervienen en la muerte materna, como las prácticas y representaciones en torno a la maternidad, las relaciones intra e interfamiliares y la presencia de diferentes alternativas de atención médica y de otro 
FIGURA 1. Localización del estado mexicano de Chiapas y el municipio de San Juan Cancuc

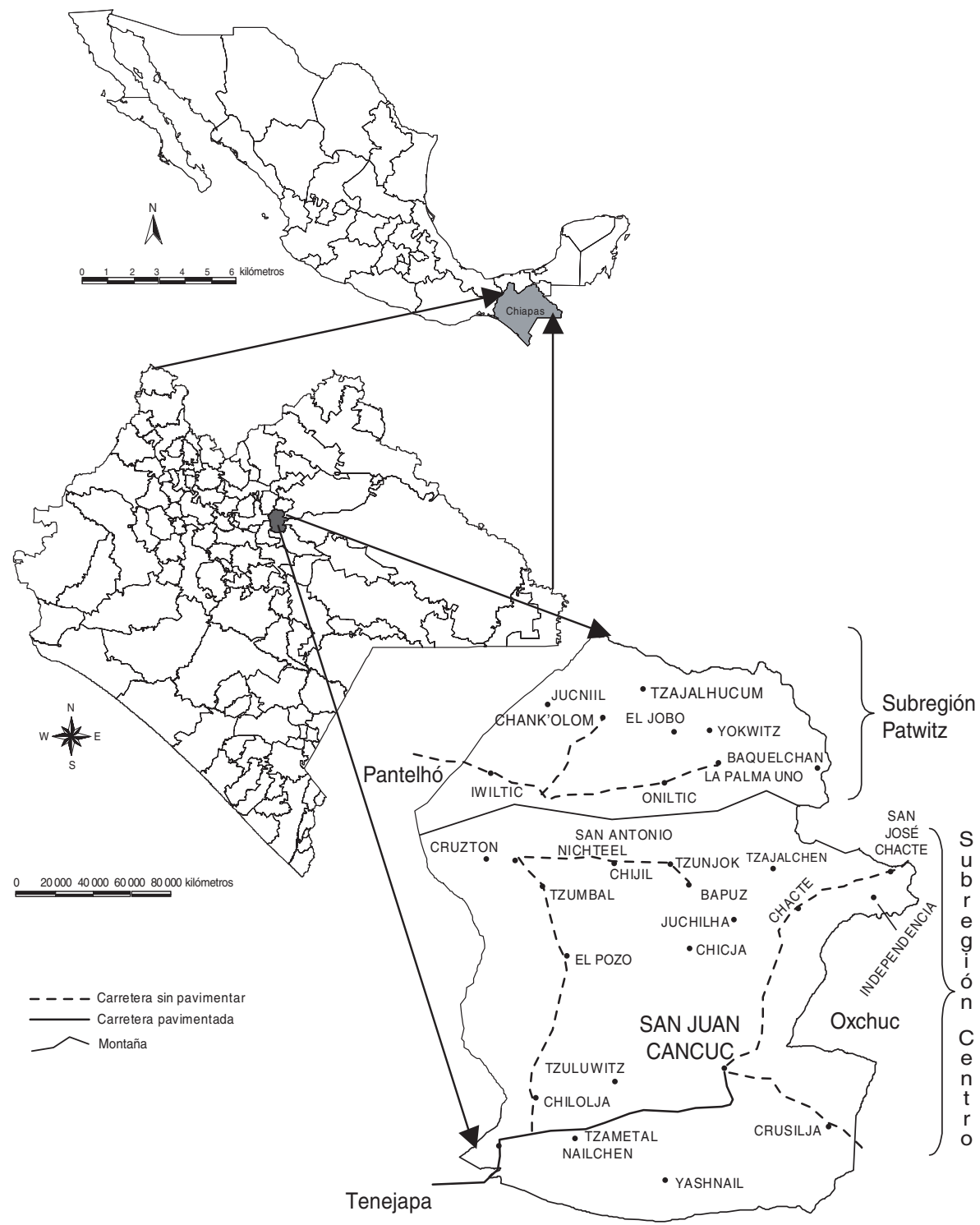

Fuente: Elaborado por Juan Sántiz Girón, ECOSUR, 2003.

tipo. El segundo trabajo (10) ofrece un análisis demográfico que pone de relieve las diferencias que se aprecian dentro del estado. Las regiones más vulnerables son Sierra, Norte, Soconusco y Altos. Señala, además, que al aplicar la tasa de mortalidad materna se advierte una tendencia contraria a la razón de mortalidad.

El tercer trabajo (11), de corte epidemiológico, toma como base el número de años en que se repiten los casos de muerte materna por municipio. Los resultados indican que hay en el estado 14 municipios de riesgo muy alto, $85,7 \%$ de ellos con un grado muy alto de marginación. ${ }^{7}$

\footnotetext{
7 Además, se clasificaron por índice de marginación, porcentaje de población indígena y presencia de programas especiales, como el Programa de Ampliación de Cobertura (PAC), el Programa de Apoyo a la Salud, Educación y Alimentación (PROGRESA) y el Programa PROSANI.
}

Esta investigación se distingue de las anteriores por su enfoque económico y sociocultural. Por tanto, la unidad de análisis fue el grupo doméstico, definido como el grupo de personas con una residencia organizada, unidos o no por lazos de parentesco, que integran una unidad de producción-consumo y de reproducción de la fuerza de trabajo (12-14). A partir de variables de ingresos y egresos se realizó un diagnóstico de la economía de la subregión tomando en cuenta todas las actividades de los grupos domésticos, además de aspectos sociodemográficos, culturales y de salud. Asimismo, en el análisis de los casos de muerte materna se destacaron como factores contribuyentes las condiciones de la economía del grupo doméstico, las relaciones de género y las reglas de residencia, parentesco y herencia.

El objetivo central que guió este proyecto fue comprender la muerte materna en el contexto del grupo doméstico. El análisis de este último permitió conocer los medios de producción limitantes de los campesinos indígenas de la subregión de Patwitz, su nivel económico y las relaciones sociales entre los miembros del grupo doméstico para la supervivencia, así como las estrategias de reproducción.

Este análisis de la muerte materna no se limita a evaluar las muertes en sí, sino que contempla las consecuencias de este suceso en los huérfanos, el viudo, la relación de la nueva esposa con los hijos y la relación del padre con los primeros hijos y con los del nuevo matrimonio. Eso significa que deben tomarse en cuenta los patrones que rigen su dinámica y que resultan de formas del ejercicio del poder, de las relaciones de género, del uso de la violencia basada en formas de autoridad, de la división sexual del trabajo, de la toma de decisiones para la distribución del presupuesto doméstico y del control de los recursos económicos (15-18).

Por consiguiente, el objetivo de este estudio ha sido conocer la situación económica y las relaciones de género según se relacionan con la muerte materna en el grupo doméstico en la sub- 
región de Patwitz, municipio San Juan Cancuc, Chiapas.

\section{MATERIALES Y MÉTODOS}

La investigación se realizó con métodos cuantitativos y cualitativos, tomando el grupo doméstico como unidad de análisis. Respecto a la parte cuantitativa, se realizó un estudio poblacional de 1039 grupos domésticos, distribuidos en las nueve comunidades que integran la subregión de Patwitz, y se procedió a obtener el tamaño de la muestra, que fue de $194 .^{8}$

Se aplicó el cuestionario a 158 grupos domésticos en siete de las nueve comunidades que integran la subregión, ${ }^{9}$ en tanto que para el análisis se establecieron cinco estratos, según el ingreso del grupo doméstico y el tamaño de la parcela de cultivo. ${ }^{10}$

El método cualitativo incluyó tres estrategias. La primera consistió en entrevistas estructuradas a personas cercanas a los casos de muerte materna registrados en el año 2002, a saber: el esposo, la suegra, la mamá, la partera y la cuñada o una mujer cercana a la fallecida. La finalidad fue conocer sus percepciones en torno a la muerte materna, la situación y posición de la mujer en el grupo doméstico, su estado de salud antes y durante las complicaciones y en el momento mismo de la muerte, además de las consecuencias económicas para el grupo y las expectativas de los viudos y huérfanos.

De las 20 entrevistas programadas se realizaron 19 , lo que constituye $95 \%$.

\footnotetext{
8 Se aplicó un muestreo aleatorio simple (19), con un nivel alfa de $95 \%, P=0,02$.

9 Las encuestas se aplicaron en las comunidades de Baquelchan (24), Chank'olom (23), Jucniil (22), Oniltic (24), La Palma (21), Tzajalhucum (20) y Yokwitz (24), lo que significa un total de 158 encuestas realizadas de las 194 programadas $(81,4 \%)$. Las encuestas fueron capturadas y analizadas, por comunidad, en el programa de análisis estadístico básico SPSS/P (20).

No se aplicó en la comunidad de El Jobo porque los habitantes de esta concentración llegan de otras comunidades de la región solo para realizar labores de siembra y para recibir el apoyo de programas gubernamentales, de modo que ya estaban incluidos en el procedimiento aleatorio. La comunidad de Iwiltic no aceptó la participación, por decisión de asamblea.

${ }_{10}$ Los estratos establecidos fueron muy bajo, bajo, medio, alto y muy alto.
}

Con autorización de los informantes se audiograbaron todas las entrevistas $\mathrm{y}$, cuando se dieron las condiciones, se videograbaron, con la finalidad de recuperar las imágenes vivas del contexto donde se produjo la muerte. En promedio, el tiempo de grabación en cada caso de muerte materna fue de cinco horas.

La base del análisis fueron las transcripciones completas de las entrevistas por un técnico experto en la lengua tseltal. Se organizó una base de datos para reconstruir cada uno de los cuatro casos de muerte materna; después se identificaron categorías y campos teóricos para el análisis y finalmente se utilizaron estos en los resultados. Las entrevistas abarcaron el periodo del 26 de marzo al 10 de julio de 2003.

La segunda estrategia cualitativa consistió en tres reuniones con parteras, una en la subregión Centro y dos en Patwitz, destinadas a evaluar su participación en el proceso reproductivo, la existencia de otros casos de muerte materna y comparar la información en torno a este suceso entre las dos regiones del municipio.

Se audiograbó cada una de las reuniones para su ulterior transcripción y análisis. Se brindó retroalimentación mediante una representación de teatro guiñol en su lengua materna, el tseltal. Estas reuniones tuvieron una duración media de tres horas.

La tercera estrategia para el análisis cualitativo, encaminada a fortalecer el proyecto y obtener un panorama más amplio del problema, consistió en la entrevista a seis informantes clave: la esposa del presidente municipal de Cancuc, el presbítero de la Diócesis de San Cristóbal, un profesor de educación primaria originario del municipio de San Juan Cancuc, el presidente del comité de educación, el esposo de la partera y el director del Instituto de Desarrollo Humano municipal. Cada una de estas entrevistas se realizó en sus centros de trabajo, con una duración promedio de dos horas.

Debe destacarse que durante todo el trabajo de campo se contó con el apoyo de un traductor indígena tseltal, capacitado en temas de salud.

\section{RESULTADOS}

\section{Conformación de los grupos domésticos: de la búsqueda de pareja a la descendencia}

A diferencia de las pautas matrimoniales que rigen en otros municipios de la región Altos, en Patwitz son las mujeres quienes eligen a su pareja. Acuden a la casa del joven a manifestar a la familia tal decisión, lo que lleva implícita su aceptación de vivir con ellos. De esa manera, se integran a ese nuevo grupo doméstico, en lo que constituye una residencia virilocal. ${ }^{11}$ Posteriormente, la pareja conformada acude a "pedir perdón" a los padres de la joven.

De acuerdo con los usos y costumbres de los tseltales, la familia del novio entrega a la de ella el "pago por la novia", que consiste en una dádiva en dinero o en especie, lo cual pone a la mujer en una posición vulnerable, ya que ni siquiera en caso de violencia física o emocional podrá ella retornar al hogar paterno, mientras su familia no pueda retribuir a la del novio lo erogado.

En esta subregión, donde el parentesco se reconoce patrilinealmente, se considera la existencia de tres clanes y linajes que debe respetarse en la búsqueda de pareja. ${ }^{12} \mathrm{Al}$ mismo tiempo, el matrimonio se produce a una edad promedio de 12 a 15 años, lo que significa que ocurren embarazos en la adolescencia, son breves los periodos entre estos y la fecundidad es alta, condiciones que tendrán repercusiones durante toda la vida de la mujer. ${ }^{13}$

En este periodo la posición de la mujer recién integrada al grupo do-

\footnotetext{
11 Residencia virilocal: regla según la cual se espera que una mujer, después de casarse, vaya a vivir con su esposo y los parientes de este.

12 San Juan Cancuc es un municipio de la región Altos de Chiapas donde aún se reconoce la existencia de tres clanes: Chejeb, Ch'ik e Ik'an. Estas clases se clasifican en linajes, los cuales incluyen determinados apellidos (Hernández, Castellano, Pats', Mexa, Bot, K'aal, Ton, Baril y Rubiz) reconocidos por las reglas de matrimonio.

13 La fecundidad correspondiente al municipio de San Juan Cancuc es de 4,4 hijos por mujer, mayor que las medias regional y estatal, de 4,3 y 3,4 , respectivamente.
} 
méstico es crítica, ya que está subordinada a la suegra y a los agnados del esposo, y es la etapa en que tiene su primer embarazo y parto. ${ }^{14}$ De esa manera, los riesgos se van acumulando conforme avanza su vida reproductiva, lo que integra un círculo vicioso de unión a edad temprana, periodos intergenésicos cortos, alimentación deficiente, atención insuficiente o nula en el embarazo, cuidados posnatales solo por cinco a ocho días e inicio inmediato de las relaciones sexuales, amamantamiento y un nuevo embarazo. ${ }^{15}$ El desgaste físico implícito se verá reflejado en los subsiguientes actos obstétricos.

Una vez que nacen los hijos, la pareja integra un nuevo grupo doméstico en residencia neolocal, es decir, con plena independencia en la producción y el consumo. Se trata de un momento crucial en la vida de la pareja, en el que la mujer tendrá que asumir todas las responsabilidades del trabajo doméstico del cuidado de los hijos y de su embarazo, parto y puerperio, además de compartir los deberes relacionados con la parcela. ${ }^{16}$

A los riesgos reproductivos se suma el hecho de que $97,7 \%$ de las mujeres hablan solo la lengua tseltal, con el aislamiento social que ello implica. Por añadidura, las mujeres no tienen derecho a la propiedad de la tierra, ya que aquí se sigue una norma de herencia patrilineal. Es decir, en la distribución de las parcelas se considera solamente a los hijos varones; si solo se tienen hijas mujeres la herencia puede derivarse a los yernos. La falta de toda posesión material entraña una grave indefensión para la mujer, ya que queda excluida de cualquier decisión sobre la venta de tierra o cualquier objeto que en algún momento podría servir para salvarle la vida.

\footnotetext{
14 Agnados: familiares emparentados de forma patrilineal.

15 Se consideran pródromos del trabajo de parto las primeras contracciones uterinas o los primeros dolores.

16 Residencia neolocal: regla según la cual después del matrimonio una pareja establece una residencia separada tanto de los familia del novio como de la novia.
}

Es en estas condiciones, propiciadoras del riesgo de muerte, como las mujeres ven transcurrir su vida reproductiva.

\section{La muerte materna en la subregión}

Los efectos de la muerte materna son de tal magnitud que repercuten durante largo tiempo, tanto en el grupo doméstico como en la comunidad y en la sociedad misma.

Inmediatamente después de la muerte sobreviene una etapa de desintegración en relación con el destino de los hijos, el cual será decidido finalmente por los familiares. Sigue a esto una etapa de adaptación, periodo en el que el grupo doméstico se habituará a la pérdida de uno de sus miembros. Los programas gubernamentales suspenden el apoyo económico al grupo doméstico de la mujer muerta en el embarazo o puerperio (fase de lactancia). Posteriormente, en una etapa de reestructuración del grupo doméstico, el cónyuge buscará una nueva pareja, mientras continúa pagando el adeudo adquirido por la compra de medicamentos e insumos para la fallecida (figura 2).

La muerte materna siempre ha estado presente en esta región. De ello dan fe tanto los testimonios de los lugareños como los datos de la encuesta. De los 158 grupos domésticos encuestados, 3,1\% manifestaron haber perdido a alguna familiar durante el proceso reproductivo - es decir, cinco muertes maternas- y la entrevista a profundidad sacó a luz un caso más, lo que constituye un total de seis defunciones adicionales, mientras que solo cuatro han sido notificadas por los sistemas oficiales de información en esta subregión en el año 2002.

Estudios referentes a Bangladesh, Egipto, Jamaica y Nueva Guinea indican que en esas naciones se notifican menos de la mitad de las defunciones maternas que realmente ocurren. Mientras que en México no se regis$\operatorname{tran} 3,5 \%$ de las defunciones de mujeres entre 15 y 49 años de edad, en el municipio chiapaneco de Chenalhó (9) se ha constatado un subregistro de
45\%. En la subregión de Patwitz, la cifra estimada es de $30 \% .{ }^{17}$

La costumbre funeraria de enterrar a los muertos en las parcelas, la larga distancia a los centros de registro en la cabecera municipal, el temor a las implicaciones morales, sociales y jurídicas y la inadecuada clasificación de las causas de muerte hacen que el subregistro prevalezca y se incremente.

En más de $50 \%$ de los casos de muerte materna no registrados, las parteras fueron llamadas en el momento del fallecimiento, en tanto que una tercera parte manifestó que la mujer fue atendida por familiares o solo por el cónyuge. En 83,3\% de los casos los familiares manifestaron que no pensaron llevarla a otro sitio a buscar atención, argumentando falta de dinero y de tiempo. Explica un entrevistado:

La gente aquí es muy pobre. No hay caminos; la gente vive lejos. Ya no alcanza el maíz; [uno] tiene que comprar. [Si a]caso lo quiere llevar su mujer a buscar doctor, no tienen dinero, tienen miedo; cuando no tenemos dinero da miedo salir. ¿Cómo va a salir sin dinero? - Todo es caro, la medicina.... Si lo saca cargando, ¿a dónde va ir? ¿Quién le presta si todos son pobres? Aquí cien o doscientos pesos es mucho dinero; son como miles de pesos. [Alonso, comunidad Yokwitz.]

En el periodo de 2001-2003 se registraron cinco muertes de mujeres jóvenes, de 25 años de edad en promedio, analfabetas, que fallecieron en el hogar en compañía solamente de su esposo, quien en el momento de las complicaciones acudió a buscar la ayuda de la partera y otros familiares. Con el tiempo esta tragedia repercute en los hijos huérfanos, que en promedio son tres por mujer muerta y tienen un promedio de 5 años de edad. Algunos son adoptados por parientes del mismo clan, mientras que otros quedan en

\footnotetext{
17 El cálculo del subregistro en la subregión de Patwitz se obtuvo, según la fórmula planteada (3), con las muertes ocurridas en el periodo de 1998-2001, que no fueron notificadas por los sistemas oficiales de información. Estos datos son el resultado de la encuesta y las entrevistas a profundidad.
} 
FIGURA 2. Efecto de la muerte materna en el grupo doméstico a lo largo del tiempo en la subregión de Patwitz, municipio de San Juan Cancuc, Chiapas, México

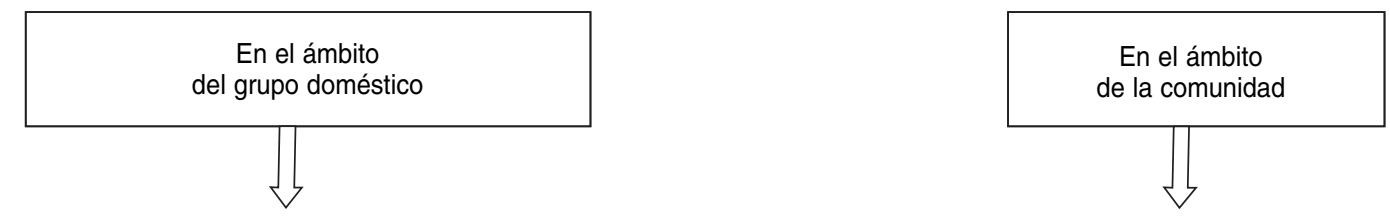

\begin{tabular}{|c|c|c|c|c|c|}
\hline $\begin{array}{l}\text { Primeros seis } \\
\text { meses después } \\
\text { de la muerte }\end{array}$ & $\begin{array}{l}\text { A un año de } \\
\text { la muerte }\end{array}$ & $\begin{array}{l}\text { A cinco años } \\
\text { de la muerte }\end{array}$ & & & \\
\hline \multirow[t]{2}{*}{$\begin{array}{l}\text { 1. Ruptura del } \\
\text { esquema familiar }\end{array}$} & \multirow{2}{*}{$\begin{array}{l}\text { 1. Cambios en las } \\
\text { responsabilidades y } \\
\text { en las actividades } \\
\text { domésticas (hija de } \\
10 \text { años) }\end{array}$} & \multirow[t]{2}{*}{$\begin{array}{l}\text { 1. Pago de deudas } \\
\text { por gastos } \\
\text { funerarios }\end{array}$} & $\begin{array}{l}\text { Primeros seis meses } \\
\text { después de la muerte }\end{array}$ & $\begin{array}{l}\text { A un año de } \\
\text { la muerte }\end{array}$ & $\begin{array}{l}\text { A cinco años } \\
\text { de la muerte }\end{array}$ \\
\hline & & & \multirow{4}{*}{$\begin{array}{l}\text { 1. Los hijos se van } \\
\text { a vivir con los } \\
\text { abuelos } \\
\text { 2. Cambios en el } \\
\text { esquema del } \\
\text { clan } \\
\text { 3. Pobreza en el } \\
\text { clan }\end{array}$} & \multirow{2}{*}{$\begin{array}{l}\text { 1. Migración de los } \\
\text { hijos }\end{array}$} & \multirow{2}{*}{$\begin{array}{l}\text { 1. Presión por } \\
\text { fuentes de } \\
\text { trabajo }\end{array}$} \\
\hline \multirow[t]{2}{*}{$\begin{array}{l}\text { 2. Menor atención a } \\
\text { los hijos }\end{array}$} & \multirow{2}{*}{$\begin{array}{l}\text { 2. Crisis en la } \\
\text { economîa } \\
\text { doméstica }\end{array}$} & \multirow{2}{*}{$\begin{array}{l}\text { 2. Pago de deudas } \\
\text { por compra de } \\
\text { alimentos tras } \\
\text { pérdida de } \\
\text { cosecha }\end{array}$} & & & \\
\hline & & & & 2. Deserción escolar & $\begin{array}{l}\text { 2. Redistribución } \\
\text { de la tierra } \\
\text { (herencia) }\end{array}$ \\
\hline $\begin{array}{l}\text { 3. Mayor probabi- } \\
\text { lidad de muerte } \\
\text { en hijos menores } \\
\text { de un año }\end{array}$ & $\begin{array}{l}\text { 3. Pérdida de la } \\
\text { cosecha }\end{array}$ & 3. Nueva pareja & & & \multirow[t]{2}{*}{$\begin{array}{l}\text { 3. Cambios en el } \\
\text { esquema } \\
\text { comunitario } \\
\text { 4. Migración }\end{array}$} \\
\hline $\begin{array}{l}\text { 4. Deserción } \\
\text { escolar }\end{array}$ & \multirow[t]{3}{*}{$\begin{array}{l}\text { 4. Suspensión de } \\
\text { la beca del } \\
\text { programa } \\
\text { Oportunidades }\end{array}$} & $\begin{array}{l}\text { 4. Los hijos se } \\
\text { sujetan a las } \\
\text { decisiones de la } \\
\text { nueva pareja }\end{array}$ & & & \\
\hline 5. Desnutrición & & $\begin{array}{l}\text { 5. Cambio de rol en } \\
\text { el núcleo familiar }\end{array}$ & & & \\
\hline $\begin{array}{l}\text { 6. Enfermedades } \\
\text { en el grupo } \\
\text { familiar }\end{array}$ & & $\begin{array}{l}\text { 6. Muerte del } \\
\text { cónyuge por } \\
\text { alcoholismo }\end{array}$ & & & \\
\hline
\end{tabular}

Fuente: Encuesta del estudio (2003).

total abandono, para vivir en condiciones de miseria, dependiendo de la comunidad para su supervivencia.

En dos de los cuatro casos de muerte materna registrados en 2002, la notificación de la causa del fallecimiento en el certificado y el acta de defunción fue incorrecta, ya que se señaló como causa básica la obstrucción placentaria (placenta previa), en tanto que los familiares de una de las difuntas manifestaron en la entrevista que falleció de hemorragia profusa en el tercer trimestre, mientras que en el otro caso el niño "venía atravesado" y no nació. Esto cambia radicalmente el dictamen del Comité Regional de Mortalidad Materna, la previsibilidad del caso y el número de huérfanos.

$\mathrm{Al}$ faltar información confiable acerca del número de muertes y de sus causas, no se establecen políticas públicas realmente acordes a la gravedad del problema.

\section{Economía de subsistencia}

La tenencia de la tierra en Patwitz es comunal. De los 158 grupos domésticos encuestados, 97,5\% manifestaron que su parcela es propia. En años recientes se ha ido incrementando el número de personas que arriendan o prestan tierras. Entre los factores por considerar están el alto crecimiento demográfico, los patrones de herencia y el bajo índice de migración, además de tener parcelas de menos de tres hectáreas situadas en zonas de bajo rendimiento agrícola, como las laderas de pendientes pronunciadas, sujetas a falta de rotación de cultivos, y la práctica de sistemas de roza, tumba y quema.

El bajo rendimiento de los cultivos y el minifundismo se reflejan en los ingresos que perciben los grupos domésticos, ya que el apoyo de programas 
gubernamentales como Procampo y Oportunidades beneficia solo a la tercera parte de los grupos domésticos encuestados, mientras que los programas de subsidio al cultivo del café, como el Fondo de Estabilización y Levantamiento de la Cosecha, alcanza apenas a $38 \%$ y $13 \%$, respectivamente. ${ }^{18}$

Aproximadamente 90,5\% de los grupos domésticos de la subregión cultivan café. El ingreso por su comercialización es un recurso anual que provee el margen necesario para absorber la pérdida generada por el consumo doméstico en los cultivos de maíz y frijol. El precio del café en la última cosecha fue de 6,50 pesos $^{19}$ por kilogramo, con una producción en la subregión de 7 quintales ${ }^{20}$ por hectárea $(\mathrm{Qq} / \mathrm{ha})$, cifra inferior a las medias regional y estatal, de 8 y 12 Qq/ha, respectivamente.

Otro ingreso de las familias de Patwitz proviene de la venta de fuerza de trabajo. Entre los 158 grupos domésticos encuestados, $15,2 \%$ trabajan como jornaleros dentro de la subregión. En general, se les contrata por uno o dos días, con una remuneración menor de dos dólares (20 pesos) al día, en la época de limpia del maíz. No existen en la subregión otras oportunidades en los sectores secundario o terciario, de manera que es la única opción también para las mujeres y los jóvenes mayores de 14 años, si bien se les remunera igual que a un varón adulto.

La suma de las tres fuentes de ingreso del grupo doméstico ofrece una idea de la situación de subsistencia en la que vive Patwitz; consiste en un ingreso promedio mensual de 1116,40 pesos mexicanos por grupo, es decir, 37,2 pesos al día (unos 3,6 dólares estadounidenses). El cuadro resulta aun más desolador si se considera que la mayor parte del ingreso, que proviene de la venta del café, se percibe sola-

\footnotetext{
18 El Programa de Apoyo al Campo (Procampo) otorga 960,00 pesos por hectárea, mientras que el programa Oportunidades pretende romper el círculo vicioso de la pobreza proporcionando apoyos efectivos a las familias para atender a sus necesidades de alimentación y nutrición, servicios básicos de salud y estímulos para que los menores continúen la educación básica.

19 Unos 0,48 dólares. El tipo de cambio vigente en junio de 2003 era de 10,35 pesos mexicanos por dólar estadounidense.

20 Es decir, 322 kilogramos. Un quintal equivale a 46 kilogramos.
}

mente durante el mes de comercialización. En otras palabras, más de la mitad del año estas personas no cuentan con dinero. Los ingresos alcanzan a cubrir solamente las necesidades básicas de los grupos domésticos.

Como se señaló antes, en la decisión sobre los egresos no tiene ninguna injerencia la mujer. Esa decisión depende forzosamente del tipo de residencia: si esta es virilocal, decidirá el padre del cónyuge; si es neolocal decidirá el esposo. En voz de un entrevistado:

Las mujeres no deciden. Si ahí comen también, tiene(n) que cumplirlo, porque ahí se toma el acuerdo. El que manda es el viejito; él va a organizar qué es lo que se compra mañana, lo que se necesita. Las mujeres no deciden nada. [Nicolás, comunidad Oniltic]

La posición de la mujer dentro del grupo doméstico es de sumisión y obediencia, lo cual subraya su condición desfavorable. No tiene voz ni voto en las decisiones sobre un ingreso al que ella contribuyó en gran medida, mientras que su situación de subordinación no le permite solicitar apoyo en momentos vulnerables, como es el proceso reproductivo.

Los egresos mensuales del grupo doméstico se destinan en $72,22 \%$ a la compra de alimentos como azúcar, aceite, sal, galletas, verduras, maíz y frijol; estos dos últimos productos alcanzan a cubrir solo tres a cinco meses la alimentación de los grupos domésticos, de manera que cada año deben gastarse 847 pesos en la compra de maíz y 812 pesos en la de frijol. Como los habitantes de la subregión no cuentan con ese dinero, un recurso para conseguir los productos consiste en pagarlos con jornadas de trabajo. Según criterios del Banco Mundial (BM), las familias que gastan más de $50 \%$ de sus ingresos deben considerarse en situación de pobreza y las que consumen todos sus ingresos, en situación de indigencia, es decir, en riesgo de perder su subsistencia (21).

Otro egreso importante son los gastos médicos, ya que $8,6 \%$ de los encuestados manifestaron que necesitan gastar en consulta privada y compra de medicamentos, como vitaminas y antibióticos. Un menor porcentaje de egresos se destina a cooperaciones comunitarias y a la compra de combustibles, herramientas y calzado, así como a la reparación de la vivienda y a la compra de materiales para la elaboración de su indumentaria.

Esta aproximación a la economía de los grupos domésticos de la subregión en los cinco estratos permite vislumbrar la realidad económica de subsistencia exigua en que estas personas viven y en la que ejercen su vida reproductiva. Los egresos mensuales superan a los ingresos, lo que obliga a los grupos domésticos a establecer estrategias de supervivencia, como la de pedir préstamos a cambio de la próxima cosecha, mantener al mínimo la alimentación, que se reduce a frijol, tortilla y café, o buscar los recursos de salud más baratos, lo que en el caso de complicaciones del embarazo o el parto puede llevar a la muerte (cuadro 1).

La contribución de la mujer a la economía del grupo doméstico es determinante para la supervivencia de este y su continuación. Dada la carga de actividades que tiene asignadas, la mujer difícilmente puede acudir en busca de atención médica en caso de molestias como dolor, hemorragia y debilidad, que asumen particular importancia durante el proceso de gestación. El que acuda a la unidad de salud significa para el grupo la pérdida de un jornal en la parcela y la acumulación de actividades intradomésticas que nadie puede o quiere realizar (figura 3).

Desde el punto de vista económico, la muerte materna significa una pérdida doble para la unidad de producción, ya que el esposo, por un lapso aproximado de un año, realizará solamente algunas actividades que les permitan sobrevivir a él y a sus hijos.

Se siente mucho si quedamos viudos, por la comida pues, por el pozol. ¿Dónde vas a encontrar? Aunque lloras, hasta te pones a moler. No vas a pedir a otra casa; no es costumbre. Tú solo a ver qué haces. ${ }^{21}$ Por la preocupación ya no se puede cortar café,

21 Pozol: bebida consistente en una mezcla de maíz molido y agua. 
CUADRO 1. Contribución de las mujeres a la economía doméstica en Patwitz, municipio de San Juan Cancuc, Chiapas, México

\begin{tabular}{|c|c|c|}
\hline Hora & Hombres & Mujeres \\
\hline 3 de la mañana & Dormidos & $\begin{array}{l}\text { Se levantan, hacen el fuego y muelen el maíz (una a } \\
\text { una hora y media) }\end{array}$ \\
\hline 4 de la mañana & $\begin{array}{l}\text { Se levantan y } \\
\text { desayunan }\end{array}$ & Hacen tortilla y café, calientan los frijoles y desayunan \\
\hline 5-8 de la mañana & Van a la parcela & $\begin{array}{l}\text { Hierven agua, cuecen el maíz, sirven el desayuno, } \\
\text { envían a los niños a la escuela, van a la parcela a } \\
\text { trabajar con el esposo y recolectan leña }\end{array}$ \\
\hline $12-13$ horas & $\begin{array}{l}\text { Descansan para comer } \\
\text { y siguen trabajando }\end{array}$ & $\begin{array}{l}\text { Descansan para comer y siguen trabajando en la } \\
\text { parcela. Buscan algunas frutas y verduras }\end{array}$ \\
\hline $15-16$ horas & $\begin{array}{l}\text { Dejan de trabajar en la } \\
\text { parcela y regresan a su } \\
\text { casa, cargando maíz, } \\
\text { frijol o leña en época de } \\
\text { cosecha }\end{array}$ & $\begin{array}{l}\text { Dejan de trabajar en la parcela y regresan a su casa } \\
\text { cargando leña o agua, aprovechan su paso por el } \\
\text { manantial para lavar la ropa }\end{array}$ \\
\hline $17-19$ horas $^{a}$ & $\begin{array}{l}\text { Llegan a su casa a } \\
\text { descansar }\end{array}$ & $\begin{array}{l}\text { Llegan a su casa, muelen el nixtamal, hacen las } \\
\text { tortillas para la cena, preparan café, tejen ropa para la } \\
\text { familia, realizan el aseo, cuecen frijol y maíz, cuidan y } \\
\text { dan de comer a los animales y van por agua }\end{array}$ \\
\hline
\end{tabular}

Fuente: Entrevistas a profundidad del estudio (2003).

a Depende de la distancia de su casa a la parcela.

FIGURA 3. Ingresos y egresos mensuales de los grupos domésticos de la región de Patwitz, municipio de Cancuc, Chiapas, México. Un peso mexicano $=0.09$ dólares, al tipo de cambio de junio de 2003.

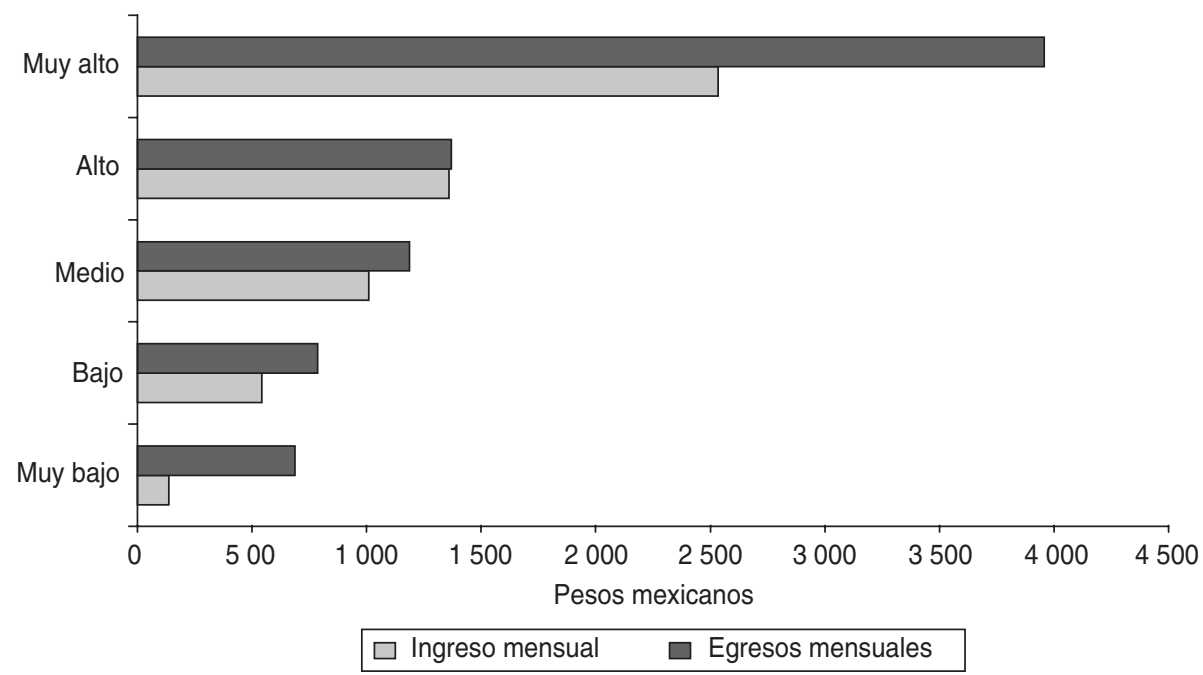

Fuente: Encuesta (mayo de 2003) y entrevistas a profundidad del estudio.

se pierde la cabeza, hasta que buscas otra mujer, al año. Ya no vas encontrar fácil; ya no viene sola. Sufrí mucho. [Diego, comunidad Oniltic.]

Estudios realizados por la OPS y el Banco Interamericano de Desarrollo
(BID) indican que es importante mejorar la salud, aun sobre los salarios individuales. Aun así, los efectos mencionados adquieren especial trascendencia entre aquellos con menos capital humano y, en consecuencia, con menores ingresos (22).

\section{Modelo de atención a las mujeres en Patwitz}

En esta subregión la atención médica institucional está a cargo de un médico, tres técnicos de salud comunitaria, un promotor de salud y nueve auxiliares de salud. Este recurso no se considera una opción viable para atender el proceso de gestación, porque, según argumentan las mujeres, la clínica está muy lejos, no es la costumbre acudir o, cuando se acude, faltan medicamentos.

La otra opción es la medicina tradicional, que en este aspecto está a cargo de las parteras. En esta subregión las hay de dos tipos: parteras particulares y parteras tradicionales. Se llama parteras particulares a familiares que tradicionalmente han atendido los partos en la familia y que, aun sin ser reconocidas como tales por la comunidad ni por los programas de capacitación institucionales, en realidad atienden la mitad de los embarazos y 45,3\% de los partos. Por otro lado, las parteras tradicionales, en número de 43 según el censo institucional, reciben del sistema información sobre los riesgos reproductivos, además de insumos para su ejercicio. Estas atienden $48,7 \%$ de los partos y la tercera parte de los embarazos.

La atención que otorgan las parteras se circunscribe a los grupos domésticos en mayor proximidad geográfica $\mathrm{y}$, en los casos de complicaciones del parto, su actuación se limita a la de espectadoras, como la de cualquier otro familiar, con tal de eximirse de eventuales responsabilidades.

A mayor abundamiento, $6 \%$ de los 158 grupos domésticos encuestados manifestaron que los partos son atendidos sin ayuda por el propio cónyuge y que durante el embarazo la mujer no recibe ningún cuidado médico. La atención por la pareja es característica de este municipio y ocasionalmente se observa también en otros municipios indígenas de la región Altos.

Esta falta de apoyo, tanto de la medicina tradicional como de la institucional, pone a las mujeres de Patwitz en alto riesgo de morir durante el embarazo, el parto o el puerperio o de sufrir lesiones incapacitantes por el resto 
de su vida, dadas las condiciones funestas del acto obstétrico.

\section{DISCUSIÓN}

Ser mujer, indígena y pobre en Chiapas es una tríada que implica un alto riesgo de morir por complicaciones del embarazo, el parto, el puerperio o el aborto. En Chiapas, 52\% de las muertes por esta causa son de mujeres indígenas. Y la población indígena representa $24,6 \%$ de la población total del estado (23).

En todos los estratos, la economía de subsistencia de los grupos domésticos depende exclusivamente del cultivo del café y de los esporádicos apoyos gubernamentales. Puesto que la medicina alópata no se considera una opción viable, por la fuerte carga que impone a los magros ingresos, resulta evidente la urgencia de definir estrategias para la atención de las mujeres gestantes.

Algunos estudios $(3,24)$ indican que $45 \%$ de los partos son atendidos por parteras no capacitadas. En el estudio presente, además de cifras similares, se encontró que la otra mitad son atendidos por familiares del mismo clan o por el cónyuge solo, porque así se evitan gastos a la precaria economía familiar. Esta situación nos pone frente a un claro panorama de muerte, si se considera que en 10 a $15 \%$ de los actos obstétricos ocurrirá alguna complicación y las mujeres no podrán recibir atención inmediata (25).

Cuando embarazos, partos, puerperios o abortos se complican, la decisión de llevar o no a la mujer a un centro de atención depende de los que detentan el poder en el grupo. En este momento crucial cuando se pone de manifiesto la falta de recursos económicos, ya que es el grupo doméstico quien debe erogar el dinero para el traslado y el tratamiento de la mujer. La comunidad participa solo como espectadora, a falta también de recursos para apoyar en esos momentos de crisis; la posibilidad de obtener un préstamo es muy remota $y$, cuando este se logra, condena al grupo a un mayor empobrecimiento, porque tardará años en cubrir la deuda contraída. A eso se debe que en $100 \%$ de los casos a las mujeres con complicaciones no se les lleve a sitios de atención apropiados. Es también el reflejo más claro de la subordinación y de la inequidad de género que prevalecen en esta zona, puesto que las mujeres no pueden decidir respecto a su salud, lo que en muchos casos las condena a morir.

Desde el punto de vista social, la mujer está prácticamente inmovilizada, porque su espacio de vida se circunscribe al grupo doméstico posmatrimonial, lo que difícilmente le permite el contacto con su familia paterna o sus amistades. A eso se suma que más de la tercera parte son monolingües y hablan su lengua materna, el tseltal, factor que les impide solicitar ayuda incluso en los estados de mayor vulnerabilidad, como el proceso gestacional, para el cual requerirían atención que difícilmente podrán buscar por sí solas $(26,27)$.

Esas condiciones se agravan por el respeto obligado a los usos y costumbres, el cual convierte la vida de las mujeres en casi una trampa. No pueden retornar a su casa paterna porque, a decir de los padres en Patwitz, son ellas quienes escogen a su pareja, o bien, como afirman los suegros: "ella vino a buscar a mi hijo". Esto, evidentemente, las coloca en una situación de desventaja. Por otra parte, la precaria situación económica determina que el "pago de la novia" no pueda resarcirse, a lo cual se añade que difícilmente la familia del esposo otorgará "permiso" para visitar a la familia paterna. De esa manera, en caso de complicaciones del proceso gestacional, la mujer estará sola y será la familia del esposo quien decida por su vida. La costumbre se vuelve así un obstáculo para que las mujeres vivan el proceso de gestación en un ambiente seguro, a salvo de la violencia y sin la violación de sus derechos más elementales.

La evaluación de la muerte materna dentro del grupo doméstico y de la comunidad nos permitió comprender que el problema no termina con las consecuencias inmediatas del deceso y de los factores que contribuyeron a él, sino que se extiende hasta las complejas secuelas emocionales de la pérdida de la hija, la esposa y la madre y persiste en el curso del tiempo con efectos tan devastadores como la muerte del recién nacido y un futuro incierto para los hijos huérfanos. La contribución de la madre al desarrollo económico y social de los hijos se verá truncada y se irá reduciendo a lo largo del tiempo, de tal forma que los integrantes de la comunidad tendrán que establecer estrategias para apoyar al grupo doméstico desintegrado, entre ellas buscar una nueva pareja para el viudo, adoptar a los huérfanos $\mathrm{y}$, a menudo, ceder para ellos sus escasos alimentos.

Como corolario se propone: 1) establecer políticas públicas que tomen en cuenta los contextos, la cultura y las condiciones socioeconómicas de los grupos domésticos, 2) analizar con un criterio multidisciplinario los planes y programas para la reducción de la muerte materna, 3) fomentar la participación comunitaria y la sensibilización de la población respecto a la magnitud del problema y 4) brindar capacitación continua y de calidad a los trabajadores comunitarios de salud y educación.

\section{REFERENCIAS}

1. World Health Organization. Children's Fund. Revised 1990 estimates of maternal mortality: a new approach, by WHO y UNICEF. Pan Am J Public Health. 1997;1:481-5.

2. Stewart MK, Stanton CK, Festin M, Jacobson $\mathrm{N}$. Issues in measuring maternal morbidity: lessons from the Philippines Safe Motherhood Survey Project. Stud Fam Plann. 1996;27(1): 29-35.

3. World Health Organization (WHO). Definitions and Recommendations: Manual of the International Statistical Classification of Dis- eases, Injuries, and Causes of Death, Vol. 1. Geneva: WHO; 1977;763-4.

4. Freyermuth EG. Morir en Chenalhó: género, etnia y generación. Factores constitutivos del riesgo durante la maternidad. [Tesis doctoral]. San Cristóbal de Las Casas (México): 
Centro de Investigaciones y Estudios Superiores en Antropología Social (CIESAS-Sureste). 2000; 234-7.

5. México, Consejo Estatal de Población en Chiapas (COESPO). Índice chiapaneco de marginación 1990-2000, 1ae ed. Tuxtla Gutiérrez (México): Talleres Gráficos de Chiapas; 2001.

6. México, Consejo Nacional de Población (CONAPO). Índices de Desarrollo Humano 2000, 1aa ed. Colección: Índices Sociodemográficos. México, D.F.: CONAPO; 2001;129.

7. México, Instituto Nacional de Geografía e Informática (INEGI). Anuario estadístico: Chiapas, México, $1^{\mathrm{a}}$ ed. México, D.F.: INEGI; 2000.

8. Freyermuth EG. Los programas de Ampliación de Cobertura y Arranque Parejo en la Vida, en los Altos de Chiapas: ¿Esfuerzos suficientes para abatir la muerte materna en el contexto multicultural? Foro Nacional de $\mathrm{Mu}-$ jeres y Políticas de Población. San Cristóbal de Las Casas: CIESAS-Sureste; 2002.

9. Freyermuth EG, Garza A. Muerte materna en Chenalhó. Informe final presentado al Programa de Salud Reproductiva de El Colegio de México (COLEM). San Cristóbal de Las Casas: CIESAS-Sureste; 1996;45-51.

10. Martínez VG, Ávila SMJ, Jáuregui DJA. La muerte materna en Chiapas: un diagnóstico desde las estadísticas vitales. Reporte del diálogo de expertos en mortalidad materna. San Cristóbal de las Casas: El Colegio de la Frontera Sur (ECOSUR); 2002.

11. México, Instituto de Salud en Chiapas (ISECH). Mortalidad materna: medidas de intervención. Reporte del diálogo de expertos en mortalidad materna. San Cristóbal de las Casas: El Colegio de la Frontera Sur (ECOSUR); 2002.
12. Oliveira O, Salles V. Grupos domésticos y reproducción cotidiana: introducción, $1^{\underline{a}}$ ed México, D.F.: El Colegio de México-Editorial Porrúa; 1989.

13. Robichaux D. Estructura y organización domésticas: una comunidad nahua en la edad industrial. En: Memoria de la Cuarta Reunión Nacional de Investigaciones Demográficas en México. Tomo 1. México, D.F.: UNAM; 1989. Pp. 745-51.

14. Arizpe SL. Parentesco y economía en una sociedad nahua, $1^{\underline{a}}$ ed. México, D.F.: Instituto Nacional Indigenista y Secretaria de Educación Pública; 1973.

15. Villasmil MC. Apuntes teóricos para la discusión sobre el concepto de estrategias en el marco de los estudios de población. Estudios Sociológicos XVI. 1998:(46):69-88.

16. Agarwal B. Negociación y relación de género: dentro y fuera de la unidad doméstica. Historia Agraria. 1999;17:13-58.

17. Cain M. Perspectives on family and fertility in developing countries. Popul Stud. 1982; 36(2):159-75.

18. Caldwell JC. Towards a restatement of demographic theory. En: Trovato F, ed. Population and society: essential readings. Oxford: Oxford University Press; 2002. Pp. 158-77.

19. Kleinbaum DG, Kupper LL, Morgenstern H. Epidemiologic research. Principles and quantitative methods. Belmont (California): Lifetime Learning Publications-Wadsworth; 1982.

20. Etxeberría J. Programación y análisis estadísticos básicos-SPSS-PC, $2^{a}$ ed. Madrid: Paraninfo; 1991.

21. Casas-Zamora JA. Salud, desarrollo humano y gobernabilidad en América Latina y el Ca- ribe a inicios del siglo XXI. Rev Panam Salud Publica. 2002;11(5-6):397-408.

22. Davedoff W, Schultz P. Wealth from health: linking social investments to earnings in Latin America. Washington, D.C.: Inter-American Development Bank; 2000.

23. México, Instituto Nacional de Geografía e Informática (INEGI). La población hablante de lengua indígena de Chiapas. México, D.F. INEGI; 2004;4-6.

24. Nazar BA. Análisis de autopsias verbales de casos de muerte materna en el estado de Chiapas, México: reporte del diálogo de expertos en mortalidad materna. San Cristóbal de las Casas: El Colegio de la Frontera Sur (ECOSUR); 2002.

25. Maine D, et al. Guidelines for Monitoring the Availability and Use of Obstetric Care. New York: UNICEF/WHO; 1997.

26. Fawcus $S$, et al. A community-based Investigation of avoidable factors for maternal mortality in Zimbabwe. Stud Fam Plann. 1996; 27(6):319-27.

27. Rowlands J. Empoderamiento y mujeres rurales en Honduras: un modelo para el desarrollo. En: León M, ed. Poder y empoderamiento de las mujeres. Santa Fe de Bogotá: Tercer Mundo Editores; 1988. Pp. 213-45.

Manuscrito recibido el 3 de marzo de 2005. Aceptado para publicación, tras revisión, el 14 de noviembre de 2005.
ABSTRACT

The household economy: a determinant of maternal death among indigenous women in Chiapas, Mexico

Key words
Objective. To assess the determining role of financial situation and gender relations on maternal mortality among Indigenous women in Chiapas, Mexico.

Methods. A quantitative/qualitative study was performed by means of a survey of 158 families, as well as in-depth interviews of persons linked to cases of maternal death, community leaders from throughout the region, and focal groups composed of traditional birth attendants.

Results. Decision-making surrounding women's health within the household is a critical problem because it is entirely in the hands of the husband and his relatives. In cases of high-risk pregnancy or birth, options for seeking care outside the community become limited, so that $48.7 \%$ of all obstetric cases are assisted by traditional birth attendants, $45.3 \%$ by relatives, and $6 \%$ by the mate. The problem is compounded by the high level of marginalization and very low human development index that characterize the region under study, by women's exclusion from the ownership of goods, including land, and by the fact that $97.7 \%$ of women only speak indigenous languages.

Conclusions. Gender inequities within Indigenous families, together with a household economy that does not cover the basic necessities, are among the factors that keep women from receiving the necessary care during their reproductive processes. Because of the low socioeconomic status these women have, decisions surrounding care during pregnancy, birth, and the puerperium take a large toll on their health and their lives.

Maternal mortality, economics, gender relations, midwives, Mexico. 